\title{
An Investigation on Autonomic Effects by Using PR Intervals
}

\author{
TW Shen, YT Tsao
}

Tzu Chi University, Hualien, Taiwan

\begin{abstract}
Many experts investigated the relations between the heart and the automatic nervous system (ANS). Most commonly, physicians nowadays use the heart rate variability $(H R V)$ to observe ANS more than others.

Instead of previous HRV method [3], the aim of this research is to find additional ANS indicator(s) for distinguishing the ANS related poses, which are resting pose and $90^{\circ}$ head-up tilt. For the result, we surprisingly discovered that the mean of PR intervals increased for 27 out of 31 persons who tilted their heads, which is better than the traditional HRV analysis. Hence, our experiment confirmed that PR interval is a potential indicator to contribute on the pacemaker algorithm, doctor diagnosis, $H R V$ analysis, and ANS physiological modelling.
\end{abstract}

\section{Introduction}

The heart is innervated by both divisions of the autonomic nervous system (ANS), which can modify the rate of the contraction, even though nervous stimulation is not required to initiate contraction [1]. Many experts are looking for the relations between the heart and the automatic nervous system (ANS). Most commonly, physicians nowadays use the heart rate variability (HRV) to observe ANS more than others. Heart rate variability, derived from the electrocardiogram (ECG), is a measurement of these naturally occurring, beat-to-beat changes in heart rate.

However, the PR interval represents the atrioventricular conduction time (AVCT), and the AVCT is coupled to the sympathetic / parasympathetic activities of the ANS [8]. Hence, the importance of PR-interval studies offers a better knowledge of the neural activity in the field of pacemaker's design and ANS study. Unfortunately, little is known about the PR autonomic modulations and the problem of estimation has been rarely addressed [2-3]. The major reason, according to HRV guidelines [3], is that precise location of a $\mathrm{P}$ wave fiducial point is very hard to achieve in surface ECGs.

In order to overcome the problem, a method is provided to obtain a location of a $\mathrm{P}$-wave fractal point in surface ECGs recorded with the current technology. In addition, the research investigated PR interval analysis and compared with ordinary HRV analysis by applying the same $90^{\circ}$ head-up tilt experiment.

In general, the frequency response of HRV is divided into three standard segments: high frequency ( $\mathrm{HF}$ - about $0.25 \mathrm{~Hz}$ ), low frequency (LF - about $0.1 \mathrm{~Hz}$ ), and very low frequency (VLF $-0.04 \mathrm{~Hz}$ to $0.13 \mathrm{~Hz}$ ). Malik et al. [3] indicated that the ratio of LF and HF power changes when subjects moved from resting to $90^{\circ}$ head-up tilt position. Our aim is mainly observe PR interval variability and search for significance on ANS regulation mechanism.

\section{Methodology}

\section{$P, Q, R, S$, and $T$ point detection}

Several digital signal processing technologies were utilized on raw ECG signals to detect PQRST fiducial points, including digital filtering, Pan and Tompkins method, first derivative ECG method (i.e., dECG) [9], and the zero-crossing method. After those steps, fiducial points are correctly detected and several interested ECG features, such as RR, PR, and PP intervals, may be able to investigate.

In order to accomplish ECG analysis, such as HRV and $\mathrm{PR}$ analysis, it is obvious that the $\mathrm{R}$ point is the major landmark which needs to be detected first. After digital filtering [4], a reliable, real-time QRS detection algorithm [5] is essential to apply. Pan and Tompkins method [5] was used in this research to determine all the $\mathrm{R}$ points in order to calculate R-R intervals.

Once the $\mathrm{R}$ point is found, the $\mathrm{Q}$ and $\mathrm{S}$ points are limited within the $150 \mathrm{~ms}$ period which is centered by the $\mathrm{R}$ point. In addition, the $\mathrm{T}$ wave is complete within a 400 ms period backward from the $R$ point, and the $P$ wave is a $200 \mathrm{~ms}$ advance from the $\mathrm{R}$ point. By using these statistical data with the first derivative ECG, the P, Q, S, and $\mathrm{T}$ points can be detected by searching minimum (valley) or maximum (peaks) of all the zero-crossing points within the certain window period $\left[t_{\text {left }}: t_{\text {right }}\right]$. For example, to detect $\mathrm{P}$ points, $t_{\text {left }}$ and $t_{\text {right }}$ were set at 
$200 \mathrm{~ms}$ and $40 \mathrm{~ms}$ advance from $\mathrm{R}$ points. The details are described as following equations:

$$
\begin{aligned}
& {\left[\begin{array}{ll}
x & y
\end{array}\right]_{Q, S}=\min _{\mathrm{Q}, \mathrm{S} \text { points }}\left\{E C G\left(\operatorname{find}\left(\operatorname{dECG}\left[t_{\text {left }}: t_{\text {right }}\right]=0\right)\right)\right\} .} \\
& {\left[\begin{array}{ll}
x & y
\end{array}\right]_{P, T}=\max _{\mathrm{P}, \mathrm{T} \text { points }}\left\{E C G\left(\operatorname{find}\left(\operatorname{dECG}\left[t_{\text {left }}: t_{\text {right }}\right]=0\right)\right)\right\} .}
\end{aligned}
$$

where $E C G(t)$ is the de-noised ECG waveform, and $d E C G(t)$ is the first derivative of the $E C G(t)$ waveform. $d E C G(t)$ combines with zero-crossing method to detect PQST points as shown in fig.1. Then fig.2 showed that the PQRST points were correctly found.

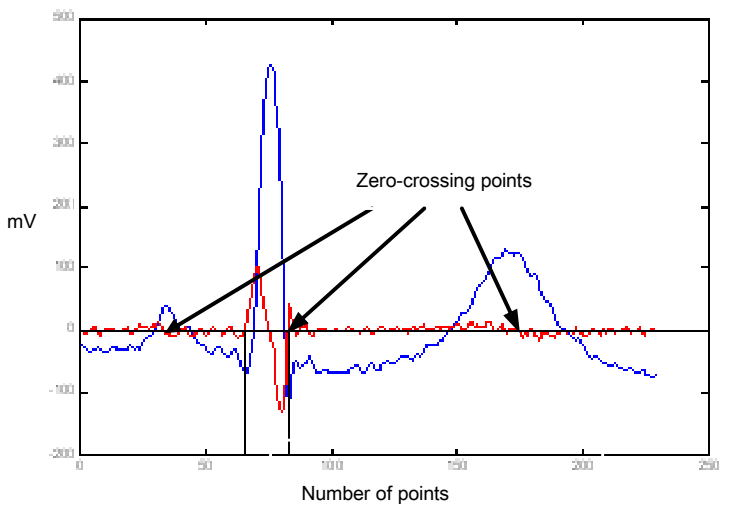

Figure 1. PQST detection by applying dECG and zerocrossing method.

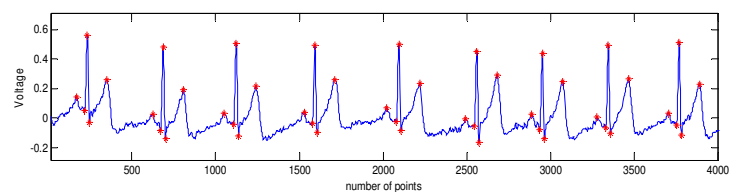

Figure 2. PQRST points are correctly detected.

For persons who have unapparent $\mathrm{P}$ waves, after removing baseline wander and other interference, the $\mathrm{P}$ waves can be enhanced by equation:

P-wave enhancement $=\sqrt{I^{2}+I I^{2}+I I I^{2}}$

where I, II, III and represent ECG lead-I, II, and III respectively.

\section{RR tachogram and PR tachogram}

Heart rate variability (HRV) is the variability of RR intervals. In this research, RR tachogram described as the five-minute (short-term) continuous series of $R R$ intervals. The tachogram was re-sampled at $2.5 \mathrm{~Hz}$ with linear interpolation and used the fast Fourier transforms (FFT) to get the frequency response to analyze.

As mentioned above, the PR interval is an important factor to evaluate automatic nervous system. However, in our research, the PR interval is defined as the temporal distance between $P$ and $R$ points. It has to be noticed that the definition of PR interval is different from the clinical description. This research not only analyzed the mean and the standard deviation of PR interval directly but also evaluated the so-called PR tachogram in order to observe the problem by using both frequency and time aspects. For PR tachogram, we used PP (time) intervals as the $\mathrm{x}$ coordinates and PR intervals as y coordinates. Like HRV, the re-sampling rate is also at $2.5 \mathrm{~Hz}$ with linear interpolation. Finally the RR tachogram and PR tachogram are plotted together in fig. 3. Their frequency power spectra are also demonstrated in fig. 4 .

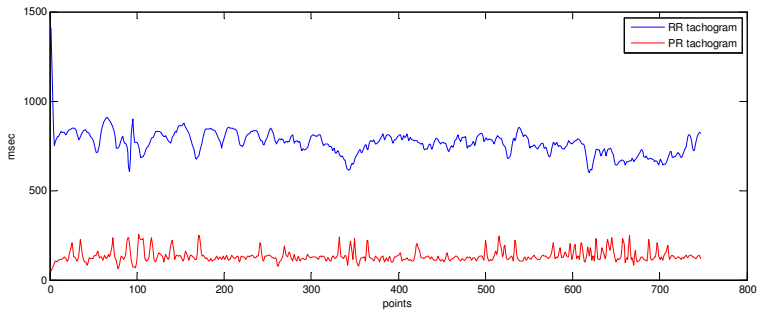

Figure 3. RR tachogram (upper) and PR tachogram (lower).
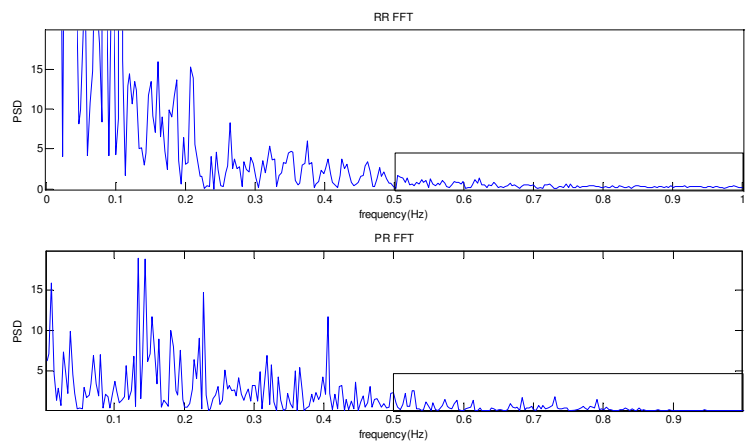

Figure 4. The frequency power spectra of RR tachogram (upper) and PR tachogram (lower)

HRV analysis and PR analysis

There are two kinds of HRV analysis: time-domain and frequency-domain. The time domain features include the mean and standard deviation (SDNN) of the RR intervals. There are also other commonly used parameters such as NN50 and pN50. The NN50 is the number of consecutive RR intervals differing more than $50 \mathrm{~ms}$. The pNN50 is the percentage value of NN50 intervals [3]. In the frequency-domain analysis, features are obtained from the power spectrum of the RR series. In general, fast Fourier transform (FFT) and autoregressive (AR) model are applied on RR tachogram to obtain the frequency power spectra. The frequency bands are divided into very low frequency (VLF), low frequency (LF), and high frequency (HF) bands. The most common frequencydomain parameters include the powers of VLF, LF, and $\mathrm{HF}$, and the LF to HF ratio [3]. Hence, the energy in the certain frequency bands can be calculated by sum of the area under the energy spectral density curve.

Similarly, for PR analysis, the time domain features include the mean and standard deviation of the PR intervals, and the frequency domain features contain the 
powers of VLF, LF, and HF, and the LF to HF ratio. All definitions of frequency bands inherited from HRV analysis.

\section{Relations between interval features by using linear regression method}

ECG signals and HRV analysis enclose so much physical information. Each amplitude, temporal distance, or frequency band contains important massages from the heart. However, the relations between six intervals, including RR, PR, RP, PP, TR, and QT intervals, are rarely discussed. Hence, our research investigated the relations by applying linear regression method. The equation is listed as follows,

$$
y=p_{1} x+p_{0}
$$

$p_{1}$ presents the slope between two variables, and it also presents the linear regression. $p_{0}$ is a bias. If the absolute value of $p_{1}$ is close to 1 , the two variables are more related. In addition, the linear regression can be used to find the correlation coefficient. Figure 5 displayed the two intervals (RR interval versus RP interval) which are highly correlated during each one of ECG cycle with the equation $y=1.0153 x-143.5928$. Nevertheless, the RR interval and PR interval are almost no correlated during each ECG cycle.
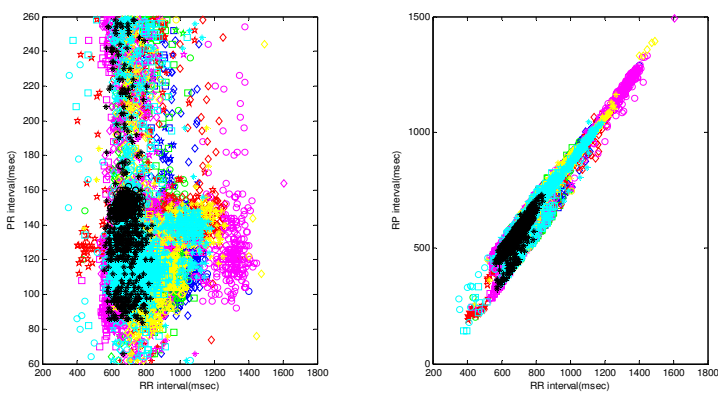

Figure 5. Linear regression: (left) RR intervals vs. PR intervals, $p_{1}=-0.085$; (right) RR intervals vs. RP intervals, $p_{1}=1.0153$.

\section{Voting system for classification}

The voting system is designed to fuse the ECG features in order to accurately distinguish rest from $90^{\circ}$ head-up tilt. The voting system involved five features which are the mean of RR intervals, the ratio $\mathrm{LF} / \mathrm{HF}$ by AR model, the ratio LF/HF by FFT model, the mean of PP intervals, and the mean of TR intervals to determine whether the person tilt up or not.

\section{Results}

Our goal of this research is to find significant ANS indicators in order to distinguish two sympathetic / parasympathetic related poses. The experiment collected lead-I and lead-III ECG signals from 31 young normal healthy subjects at the sampling rate $500 \mathrm{~Hz}$. Two five- minute (short-term [3]) ECG signals were recorded both at rest and during $90^{\circ}$ head-up tilt. An ECG data acquisition unit (BIOPAC Student Lab PRO system MP35 with software) is used to measure above ECG signals. Table 1 shows the general statistic data from 31 subjects.

Table 1. General statistic data from 31 normal subjects

\begin{tabular}{|l|c|}
\hline Age(years) & $22.7 \pm 2.1$ \\
\hline Height $(\mathrm{cm})$ & $166.4 \pm 8.4$ \\
\hline Weight $(\mathrm{kg})$ & $59.6 \pm 11.1$ \\
\hline
\end{tabular}

The re-sampling process of RR series can cause tachogram distortion. The PRD (percent root-meansquare difference) was estimated the effect. After calculation, the average of PRD is 0.0098 all RR tachograms. Table2 showed outcomes of all parameters related with HRV and six intervals which mentioned in 2.4 .

Table 2. HRV parameters and time intervals

\begin{tabular}{|c|c|c|}
\hline \multirow[t]{2}{*}{ Parameters } & \multicolumn{2}{|c|}{ Mean \pm SD } \\
\hline & rest & tilt \\
\hline HR (beats/minute) & $76.2 \pm 12.5$ & $79.3 \pm 13.2$ \\
\hline RMSSD* ${ }^{*}(\mathrm{~ms})$ & 55.33 & 49.25 \\
\hline NN50* (count) & 74.68 & 53.71 \\
\hline pNN50* $\quad(\%)$ & 22.07 & 15.69 \\
\hline RR interval (msec) & $817.3 \pm 149.9$ & $787.3 \pm 149.5$ \\
\hline PR interval (msec) & $131.3 \pm 11.8$ & $137.7 \pm 14.1$ \\
\hline $\begin{array}{l}\text { PR interval (msec) - } \\
\text { after upper and lower } \\
10 \% \text { outliers were } \\
\text { omitted. }\end{array}$ & $127.02 \pm 11.8$ & $131.96 \pm 15.5$ \\
\hline $\mathrm{RP}$ interval (msec) & $685.9 \pm 151.3$ & $649.7 \pm 154.3$ \\
\hline PP interval (msec) & $817.3 \pm 149.9$ & $787.4 \pm 149.5$ \\
\hline RT interval (msec) & $242.8 \pm 28.3$ & $239.8 \pm 25.5$ \\
\hline QT interval (msec) & $315.8 \pm 27.3$ & $312.7 \pm 24.3$ \\
\hline LF/HF(AR model) & $4.97 \pm 5.27 *$ & $9.5 \pm 20.27 *$ \\
\hline LF/HF(FFT model) & $\begin{array}{l}2.53 \pm 2.38 * \\
2.94 \pm 2.51\end{array}$ & $\begin{array}{l}3.14 \pm 4.86^{*} \\
3.55 \pm 4.47\end{array}$ \\
\hline
\end{tabular}

The symbol * indicates the listed value which calculated by using the software developed from Niskanen et al. [6]. Other values came from the MATLAB program we developed.

The correlation coefficients between six interval features, including RR, PR, RP, PP, TR, and QT intervals, are listed at Table 3 . Unlike high correlation between RR and PR intervals, RR intervals and PR intervals have almost no correlation from globe scale (31 subjects).

Table 3. Correlation coefficients between six interval features for total of 31 subjects

\begin{tabular}{|r|r|r|r|r|r|r|}
\hline & RR & PR & RP & PP & RT & QT \\
\hline RR & 1 & -0.085 & 1.0153 & 1.0073 & 0.1135 & 0.1079 \\
\hline
\end{tabular}




\begin{tabular}{|c|c|c|c|c|c|c|}
\hline PR & -0.085 & 1 & -0.295 & -0.174 & -0.101 & -0.043 \\
\hline RP & 1.0153 & -0.2947 & 1 & 0.9838 & 0.1070 & 0.1009 \\
\hline PP & 1.0073 & -0.1735 & 0.9838 & 1 & 0.1044 & 0.0975 \\
\hline RT & 0.1135 & -0.1005 & 0.1070 & 0.1044 & 1 & 0.5602 \\
\hline QT & 0.1079 & -0.0428 & 0.1009 & 0.0975 & 0.5602 & 1 \\
\hline
\end{tabular}

For the local scale, by observing the same person with different ANS-related poses, PR intervals may actually increase when RR intervals decreased as shown on Fig. 6.
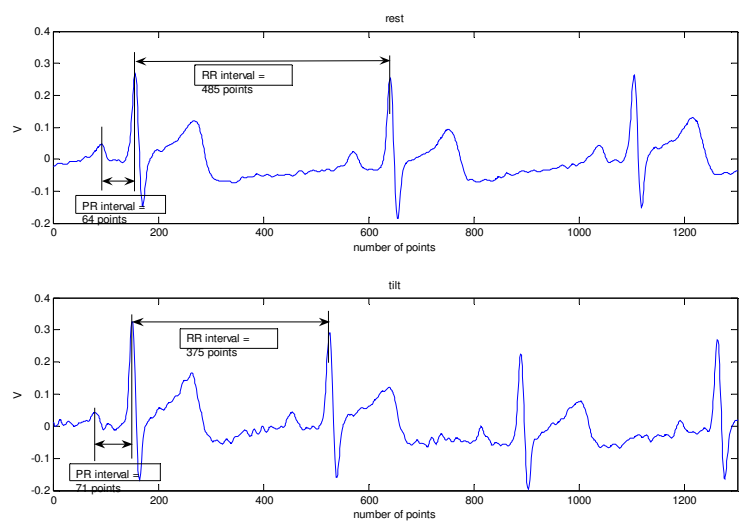

Figure 6. PR intervals may actuality increase when RR intervals decreased.

Again, the previous research [3] used the ratio LF/HF to distinguish two poses. For our experiment data, the $\mathrm{LF} / \mathrm{HF}$ values of both AR and FFT models calculated by Niskanen's software [6] provided 58.06\% (18/31) accuracy to classify two poses. By comparison, our program provides $67.74 \%$ (21/31) accuracy for distinguishing poses. According to the above results, the ratio of LF/HF from short-term HRV may not classify two events well. In addition, a five-feature voting system (mentioned in session 2.5) was developed to improve the performance. The results showed that the voting model has $77.4 \%$ accuracy. Finally, by applying the same database, we surprisingly discovered that the mean of PR intervals increased for $87.1 \%$ people who tilted their heads. However, there is no significance that was found on standard deviation and frequency spectrum of PR tachogram. Table 4 compared all performances.

Table 4. Accuracy for classifying poses.

\begin{tabular}{|l|l|l|}
\hline & Number of exception & accuracy \\
\hline HRV software [6] & 13 & $58.06 \%$ \\
\hline Our HRV program & 10 & $67.74 \%$ \\
\hline VOTING & 7 & $77.4 \%$ \\
\hline Mean of PR intervals & 4 & $87.1 \%$ \\
\hline
\end{tabular}

\section{Discussion and conclusions}

Based on our experiment, standard HRV analysis [3] seems not the only choice to distinguish from resting position to $90^{\circ}$ head-up tilt position, especially for shortterm HRV. Hence, we make a hypothesis to explain why the PR interval influenced more than others - The head- up tilt position provides sympathetic stress, so the heart rate increases. However, the parasympathetic sympathetic system would like to restrain in the current state as a biofeedback, so the PR intervals prolong and tied to decrease the heart rate.

Our future work will study more on PR interval variability to obtain further knowledge on automatic nervous system. Moreover, the developed technologies may be contributed on the pacemaker algorithm, doctor diagnosis, HRV analysis, and ANS physiological modelling.

\section{Acknowledgements}

This work was supported in part by the Taiwan National Science Council under Grant NSC-94-2218-E320. Thanks Professor Willis J. Tompkins who gave the suggestions on $\mathrm{P}$ wave enhancement.

\section{References}

[1] Sherwood L. Human Physiology From Cells to Systems, $3^{\text {rd }}$ ed.. Wadsworth Publishing Inc., 1997:282-290.

[2] Cabasson A, Meste O, Blain G, Bermon S. A New Modeling of the Overlapping $\mathrm{T}$ Wave for the efficient Estimation of the P-R Intervals during Exercise and Recovery, Proceedings of the 29th Annual International Conference of the IEEE EMBS 2007:616-619.

[3] Malik M, et al., Guidelines: Heart Rate Variability, Standards of measurement, physiological interpretation, and clinical use. European Heart Journal, 1996:17:354-381.

[4] Shen TW, Shan HP, Lin CH, Ou YL. Detection and Prediction of Sudden Cardiac Death (SCD) for Personal Healthcare. 29th IEEE Engineering in Medicine and Biology Society, 2007: 2575-2578.

[5] Pan J, Tompkins WJ, A real-time QRS detection algorithm. IEEE Trans. Biomed. Eng., BME-32(3):230236, 1985.

[6] Niskanen JP, Tarvainen MP, Perttu O. Ranta-aho. Software for advanced HRV analysis. University of Kuopio Department of Applied Physics Report Series ISSN 07884672, 2002.

[7] Teich MC, Lowen SB, Jost BM, Vibe-Rheymer K. Heart Rate Variability: Measures and Models. Electrical and Computer Engineering, Boston University, 2000:1:7:1-84

[8] Hexamer M, Nagel M, Werner J. Rate-responsive pacing based on the atrio-ventricular conduction time: Comparison of different algorithms. Medical Engineering \& Physics 2006:28:894-904.

[9] Kamath C, Ananthapadmanabhayuyu TV, Modeling QRS Complex in dECG. IEEE Trans. Biomed. Eng., 2007: 54: $1: 156-8$

Address for correspondence

Tsu-Wang Shen, Ph.D.

Department of Medical Informatics

Tzu-Chi University

701, Sec. 3, Jhongyang Rd.

Hualien, 97004, Taiwan

tshen@mail.tcu.edu.tw 